Journal für Mobilität und Verkehr

ISSN 2628-4154

www.dvwg.de

\title{
Auswirkungen von COVID-19 auf das Arbeiten von Zuhause - eine Analyse auf Basis der Da- ten des Deutschen Mobilitätpanels
}

\author{
Anna Reiffer*, Miriam Magdolen, Lisa Ecke, Peter Vortisch \\ Siehe AutorInnenangaben
}

\section{Abstract}

In diesem Paper wird eine Analyse der Auswirkungen von Home Office und der Einflüsse auf die Entscheidung, von zu Hause aus zu arbeiten vorgestellt. Dank des Paneldesigns liefert das Deutsche Mobilitätspanel einzigartige Daten von Personen, die vor und während der COVID-19-Pandemie teilgenommen haben. Unsere Ergebnisse zeigen, dass die die Pandemie das Pendelverhalten stark beeinflusst hat und die Veränderungen teilweise auch zukünftig bleiben werden.

\section{EINLEITUNG}

Der Ausbruch des Coronavirus (COVID-19) im Jahr 2019 in Wuhan, China (WHO, 2020) und die anschließende Ausrufung einer weltweiten Pandemie hatten erhebliche Auswirkungen auf das tägliche Leben und Verhalten der Menschen. Um die Verbreitung des Virus einzuschränken, haben viele Regierungen verschiedene Maßnahmen angeordnet: Vorschriften, in der Öffentlichkeit eine Maske zu tragen, Aufforderungen, zu Hause zu bleiben, die Schließung von Geschäften und Restaurants sowie von Schulen und Arbeitsstätten. Aufgrund des veränderten Aktivitätsniveaus und Sicherheitsempfindens konnten erhebliche Veränderungen im Mobilitätsverhalten beobachtet werden. Die Arbeit von Molloy et al. (Molloy et al., 2021) zeigt allerdings, dass sich die meisten dieser Veränderungen mit zunehmender Lockerung der Maßnahmen wieder zurückentwickeln. Dies gilt jedoch wahrscheinlich nicht für die Veränderungen der Einstellung und des Verhaltens bei der Arbeit von zu Hause. Die COVID19-Pandemie hat ArbeitgeberInnen und ArbeitnehmerInnen gezwungen, ihre persönliche Einstellung zur Heimarbeit zu überdenken, da Interaktionen während des Pendelns und am Arbeitsplatz mit einem hohen Infektionsrisiko in Verbindung gebracht wurden. Die daraus resultierenden Verände- rungen im Pendelverhalten sind in verschiedenen Studien beschrieben (Beck et al., 2020; de Haas et al., 2020; Hiselius and Arnfalk, 2021; Shamshiripour et al., 2020). Was die langfristigen Auswirkungen der Pandemie auf die Einstellung zur Heimarbeit betrifft, so berichten viele Studien, dass viele Erwerbstätige auch in Zukunft die Möglichkeit zur HO-Nutzung haben möchten (Beck et al., 2020; de Haas et al., 2020; Kolarova et al., 2021). Obwohl die technischen Vorraussetzungen für die Heimarbeit schon länger gegeben sind, arbeiteten vor Beginn der COVID-19 Pandemie die meisten Erwerbstätigen vor Ort und nicht zuhause (European Foundation for the Improvement of Living and Working Conditions, 2018). Das liegt vor allem daran, dass nicht jede Tätigkeit von zuhause durchgeführt werden kann (Mokhtarian and Salomon, 1996a, 1996b). Vor der COVID-19-Pandemie haben vor allem hochqualifizierte Fach- und Führungskräfte zuhause gearbeitet (European Foundation for the Improvement of Living and Working Conditions, 2010; Felstead et al., 2002), die ein hohes Maß an Autonomie in Bezug auf ihre Arbeit haben (Clear and Dickson, 2005).

Mit diesem Paper ergänzen wir diese Erkenntnisse durch Analysen basierend auf Daten des Deutschen Mobilitätspanels (MOP). Durch die Verwendung von Daten aus Kohor-

\footnotetext{
* Korrespondierende Autorin
} 
ten, die nicht nur während der Pandemie, sondern auch in den Jahren 2018 und 2019 berichtet haben, ist es möglich, Verhaltensänderungen bei denselben Personen zu erfassen. So können wir weitere Erkenntnisse über die Auswirkungen der COVID-19-Pandemie auf Home-Office-Nutzung gewinnen und was dies für VerkehrsplanerInnen und politische EntscheidungsträgerInnen bedeutet. In diesem Paper geben wir zunächst einen kurzen Überblick über das MOP und die von uns verwendeten Daten. Wir präsentieren die wichtigsten Erkenntnisse aus den Befragungskohorten, die 2018 und/oder 2019 und 2020 teilgenommen haben, in Bezug auf Home-Office $(\mathrm{HO})$ und das damit verbundene Verkehrsverhalten. Anschließend leiten wir mithilfe eines linearen Regressionsmodells Effekte der Pandemie auf das Pendelverhalten ab. Darüber hinaus präsentieren wir die Ergebnisse von Logit-Modellen zu Home-Office-Entscheidungen. Abschließend diskutieren wir die Ergebnisse und schließen den Beitrag mit Implikationen für Verkehrsplaner und politische EntscheidungsträgerInnen im Hinblick auf zukünftige HO-Nutzung.

\section{DATEN}

Für unsere Analysen verwenden wir die Daten des Deutschen Mobilitätspanels (MOP). Das MOP erhebt seit 1994 Daten zum Mobilitätsverhalten der deutschen Bevölkerung. Ungefähr 1.800-2.000 Haushalte mit 3.000-3.400 Befragten ab zehn Jahre nehmen jedes Jahr am MOP teil. Der jährliche Erhebungszeitraum liegt im Herbst und schließt alle Feiertage aus, um das alltägliche Mobilitätsverhalten bestmöglich zu erfassen. Die TeilnehmerInnen werden gebeten, ein Wegetagebuch zu führen, das Informationen über alle von innen an sieben aufeinanderfolgenden Tagen unternommenen Wegen enthält, inklusive Entfernungen, Verkehrsmittel, Zwecke sowie Start- und Ankunftszeiten. Darüber hinaus werden soziodemografische Informationen über die Teilnehmerlnnen (z. B. Beschäftigungsstatus, Geschlecht, Alter) und Merkmale zur Mobilität erhoben. Die Erhebung wird im Auftrag und mit Mitteln des Bundesministeriums für Verkehr und digitale Infrastruktur (BMVI) durchgeführt, wobei das Institut für Verkehrswesen am Karlsruher Institut für Technologie (KIT) für die Konzeption und wissenschaftliche Begleitung der Befragung verantwortlich ist (Ecke et al., 2020; Zumkeller and Chlond, 2009).

Für unsere Analyse verwenden wir Daten aus drei Jahren (2018-2020). Zwei Datensätze, die im Herbst/Winter 2018 und 2019 erhoben wurden, dienen als Referenz für die Zeit vor der COVID-19-Pandemie. Ein dritter Datensatz wurde im Herbst 2020 während der Pandemie erhoben.

Für unsere Untersuchungen verwenden wir eine Teilstichprobe, die sich auf Erwerbstätige und ihr Mobilitätsverhalten sowie der HO-Nutzung konzentriert. Wir betrachten nur TeilnehmerInnen ab einem Alter von 18 Jahren, die in Vollzeit oder Teilzeit beschäftigt sind und die während des Erhebungszeitraums keine Besonderheiten (z. B. Krankheit oder Urlaub), keinen Umzug oder Arbeitsplatzwechsel berichtet haben. Insgesamt enthalten die von uns verwendeten Daten Informationen aus 2.117 Berichten von 1.138 Befragten.

TABELLE 1 zeigt die Merkmale der Stichprobe 2020, die wir für unsere Analysen verwenden. Zum Vergleich sind auch die Statistiken der erwerbstätigen Bevölkerung von 2019 angegeben.

TABELLE 1 Merkmale der genutzten Stichprobe

\begin{tabular}{llcc}
\hline Variable & Ausprägung & Stichprobe [\%] & Erwerbstätige Bevölkerunga [\%] \\
\hline \multirow{2}{*}{ Geschlecht } & Männlich & 48.7 & 53.3 \\
& Weiblich & 51.3 & 46.7 \\
\hline \multirow{2}{*}{ Alter (Jahre) } & $<25$ & 0.6 & 9.6 \\
& $25-35$ & 11.3 & 20.0 \\
& $35-50$ & 32.0 & 32.2 \\
& $50-60$ & 40.6 & 26.9 \\
\multirow{2}{*}{ Beruflicher Status } & $60+$ & 15.5 & 11.3 \\
\hline \multirow{2}{*}{ Bildung } & Vollzeit & 76.6 & 70.8 \\
& Teilzeit & 27.4 & 29.2 \\
\hline \multirow{2}{*}{ Ökonomsicher Status } & Niedrig & 9.7 & \\
& Mittel & 28.5 & \\
\hline \hline a Statistiken zur erwerbstätigen & Bevölkerung aus dem Jahr 2019 (Situation vor der COVID-19-Pandemie) \\
(Destatis, 2019) & Niedrig & 81.8 & \\
\hline \hline
\end{tabular}


Es gilt zu anzumerken, dass die Statistiken für die erwerbstätige Bevölkerung für einige Variablen, wie den Berufsstatus, durch die COVID-19-Pandemie erheblich beeinflusst wurden. Die ungewichtete Stichprobe aus dem MOP weist Unterschiede zu den Statistiken für 2019 auf. Dies ist darauf zurückzuführen, dass jede Kohorte so rekrutiert wird, dass sie individuell repräsentativ für die Bevölkerung ist. In unserer Studie werden nur diejenigen dargestellt, die zum zweiten oder dritten Mal teilnehmen (WiederholerInnen). Für die Analysen wurden die Daten nicht gewichtet.

\section{DESKRIPTIVE ANALYSE}

In diesem Abschnitt diskutieren wir die wichtigsten Ergebnisse der deskriptiven Analyse. Wir konzentrieren uns auf wichtige Erkenntnisse darüber, wie Personen ihre berufsbedingten Wege und HO-Aktivitäten während der COVID19-Pandemie in Deutschland verändert haben. Darüber hi- naus werden Erkenntnisse zu den Einstellungen gegenüber Arbeiten im $\mathrm{HO}$ vorgestellt.

Im MOP werden die Teilnehmerlnnen gebeten, ihren HOStatus anzugeben, indem sie die Frage „Besteht die Möglichkeit, an manchen Werktagen ausschließlich von zuhause aus zu arbeiten und nutzen Sie die Möglichkeit?" beantworten, wobei eine von vier Kategorien auszuwählen ist: "Diese Möglichkeit besteht und ich nutze sie häufig (mindestens wöchentlich).", "Diese Möglichkeit besteht und ich nutze sie gelegentlich.", "Diese Möglichkeit besteht, aber ich nutze sie nicht." und "Diese Möglichkeit besteht nicht.". Um die Veränderung der HO-Nutzung während der COVID-19-Pandemie zu bewerten, vergleichen wir die gegebenen Antworten zum HO-Status. Abbildung 1 zeigt die Veränderungen des $\mathrm{HO}$-Status in den drei Jahren gleicher Teilnehmerlnnen. Die HO-Nutzung ist bereits zwischen 2018 und 2019 leicht angestiegen, wobei der Anstieg im Jahr 2020 deutlich stärker zu erkennen ist.

Abbildung 1 Veränderung der HO-Verfügbarkeit und HO-Nutzung zwischen 2018, 2019 und 2020 von Personen, die in allen drei Jahren teilgenommen haben $(\mathrm{N}=330)$

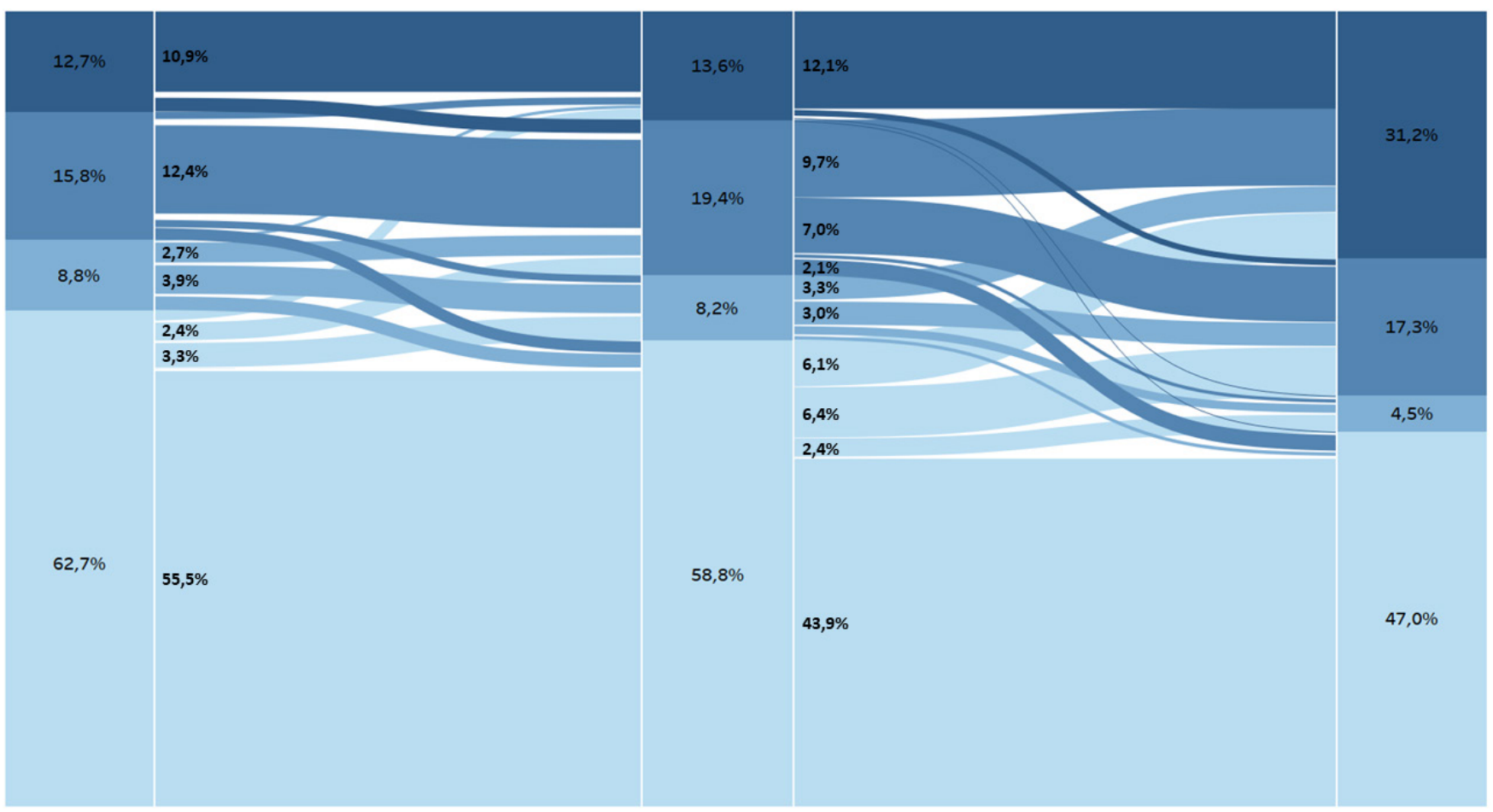

Diese Möglichkeit besteht und ich nutze sie häufig. ㅁ Diese Möglichkeit besteht und ich nutze sie gelegentlich. — Diese Möglichkeit besteht, aber ich nutze sie nicht.

Die vermehrte Nutzung von $\mathrm{HO}$ hat erhebliche Auswirkungen auf das Verkehrssystem, da das Mobilitätsniveau und insbesondere Pendlwege reduziert werden. Die Analyse des MOP zeigt den Rückgang der Wege und der zurückgelegten Entfernungen im Jahr 2020 im Vergleich zum Vorjahr. Im Durchschnitt unternahmen die Beschäftigten im Jahr 2019
15,7 Wege pro Woche und im Jahr 2020 12,1 Wege pro Woche. Bei den zurückgelegten Entfernungen ist ein Rückgang von $31 \%$ zwischen diesen Jahren zu verzeichnen. Es ist anzumerken, dass Personen, die im $\mathrm{HO}$ arbeiten können, auch unter nicht-pandemischen Bedingungen mehr Wege zurücklegen als Personen, die nicht im $\mathrm{HO}$ arbeiten können. 
Die Gesamtzahl der Wege und der zurückgelegten Entfernungen für Arbeits- und Geschäftszwecke innerhalb einer Woche ist zwischen 2019 und 2020 erheblich zurückgegangen. Die durchschnittliche Anzahl Wege zu Arbeitszwecken sank von 4,0 Wege (2019) auf 3,3 Wege (2020). Für geschäftliche Zwecke ist ebenfalls ein Rückgang der durchschnittlichen Anzahl der Wege von 1,4 Wegen (2019) auf 0,9 Wegen zu erkennen.

In der MOP-Umfrage wurden die Teilnehmerlnnen auch gefragt, wie sie die Arbeit im $\mathrm{HO}$ bewerten und was ihre ArbeitgeberInnen tun, um $\mathrm{HO}$ zu ermöglichen (um dadurch den engen Kontakt zu unterbinden). Ein wichtiges Ergebnis ist, dass die meisten TeilnehmerInnen der Umfrage der Arbeit im $\mathrm{HO}$ allgemein positiv gegenüberstehen. Abbildung 2 zeigt die Intensität der HO-Nutzung in Abhängigkeit der Einstellung der Befragten zum $\mathrm{HO}$ und den Erwartungen der Arbeitgeberln an sie. Die meisten (76\%), die eine positive Ein- stellung zu $\mathrm{HO}$ haben, haben auch eine positive Einstellung zu einer häufigeren HO-Nutzung. Im Gegensatz dazu sehen wir, dass diejenigen, die eine negative Einstellung zu $\mathrm{HO}$ haben, diejenigen sind, die auch nicht von zu Hause arbeiten können. Nur $9 \%$ der Personen, die eine negative Einstellung zu HO haben, sind Personen, die gleichzeitig häufig von zu Hause arbeiten.

Ein interessantes Ergebnis ist, dass $68 \%$ der Personen, die geantwortet haben, dass ihre Arbeitgeberln mehr $\mathrm{HO}$ wünschen, auch diejenigen sind, die bereits häufig im $\mathrm{HO}$ arbeiten. Wenn in Zukunft mehr Arbeit von zu Hause möglich ist, könnte dies zu einer erheblichen Verringerung der Pendlerund Geschäftsreisen führen. Eine große Anzahl der Teilnehmerlnnen antwortete, dass ihre ArbeitgeberInnen auch in 2020 die HO-Regelungen nicht geändert haben. Dieser Aussage stimmen vor allem die Personen zu, die nicht im $\mathrm{HO}$ arbeiten können.

Abbildung 2 Einstellungen zur Arbeit von Zuahuse, bewertet von Arbeitnehmerlnnen (für sich selbst und ihren Arbeitgeberln; S1-S6), in Abhängigkeit der HO-Verfügbarkeit und HO-Nutzung im Jahr 2020, (S1 N=151; S2 N=175; S3 N=70; S4 N=96; S5 N=101; S6 N=218)

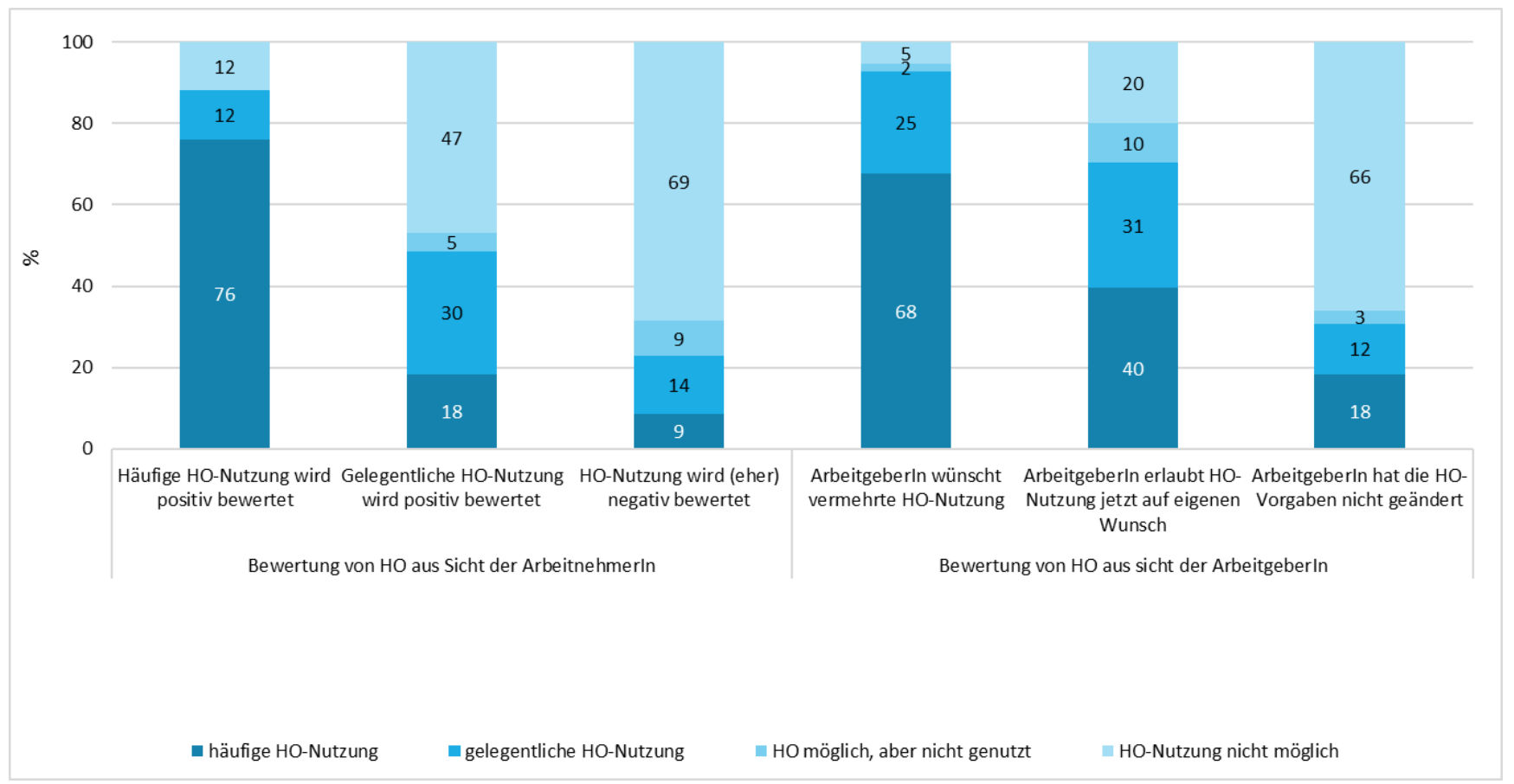

\section{STATISTISCHE MODELLIERUNG}

Um weitere Erkenntnisse über das Pendlerverhalten und das Arbeiten von Zuhause zu gewinnen, haben wir mehrere Modelle angewandt. Um die Auswirkungen der Pandemie auf das Pendlerverhalten zu analysieren, haben wir ein multiples lineares Regressionsmodell geschätzt. Darüber hinaus haben wir mit einem binären Logit-Modell bzw. einem gemischten Logit-Modell ermittelt, welche Faktoren die Möglichkeit, von Zuahuse aus zu arbeiten, beeinflussen und welche Merkmale die Entscheidung für das Arbeiten von Zuhause bestimmen.

\section{Regressionsanalyse zur Analyse des Pendlerverhaltens}

In diesem Abschnitt präsentieren wir die Analyse zum berichteten Verhalten der Erwerbstätigen, die in den Jahren 2019 und 2020 an der Erhebung teilgenommen haben, um die Auswirkungen von COVID-19 auf das Pendelverhalten zu untersuchen. Wir schließen TeilnehmerInnen aus, bei denen Werte fehlen oder die in einer der beiden Jahre 
Urlaub oder Krankheit angegeben haben oder die zwischen den beiden Berichten den Arbeitsort gewechselt haben, um andere Gründe auszuschließen, die die Anzahl der Pendelwege beeinflussen. Die verbleibende Stichprobengröße für die folgende Analyse beträgt 498. Um die Auswirkungen auf das Pendelverhalten zu messen, berechnen wir die Veränderung der Anzahl der Wege zur Arbeit in den Jahren 2019 und 2020 auf individueller Ebene. In dieser Analyse werden die soziodemografischen Merkmale der TeilnehmerInnen berücksichtigt, um Aufschluss darüber zu erhalten, wer die Zahl der Wege zur Arbeit während der COVID-19-Pandemie reduziert hat.

Abbildung 3 zeigt einen Überblick über die Veränderungen bei der Anzahl der Wege zwischen den Jahren. Insgesamt ist eine Verschiebung zu positiven Werten zu erkennen, was auf eine Verringerung der Wege zur Arbeit hindeutet. Bei Personen, die mit dem Auto zur Arbeit fahren, war die Wahrscheinlichkeit größer, dass sie ihre Wege reduzierten. Je hö- her der ökonomische Status, desto wahrscheinlicher ist ein Rückgang der Wege zur Arbeit. In Übereinstimmung mit den vorangegangenen Ergebnissen zeigt sich, dass Personen, die die Möglichkeit haben, flexibel zu arbeiten, auch eher bereit sind, seltener zu ihrem Arbeitsplatz zu fahren. Personen mit einem Hochschulabschluss reduzierten während der COVID19-Pandemie mit größerer Wahrscheinlichkeit ihre Wege zur Arbeit.

Um Beziehungen zwischen einem Merkmal und der Veränderung der Anzahl der Pendelwege zu ermitteln, führen wir eine multiple lineare Regression durch. Die Differenz der Anzahl der Wege ist dabei die abhängige Variable in dem Modell. Nach der Durchführung mehrerer Modelle wurde das endgültige Modell auf der Grundlage der Signifikanz der unabhängigen Variablen sowie des R-Quadrat-Werts ausgewählt. Das Gesamtmodell ist signifikant (<.0001). Die Ergebnisse sind in Tabelle 2 dargestellt.
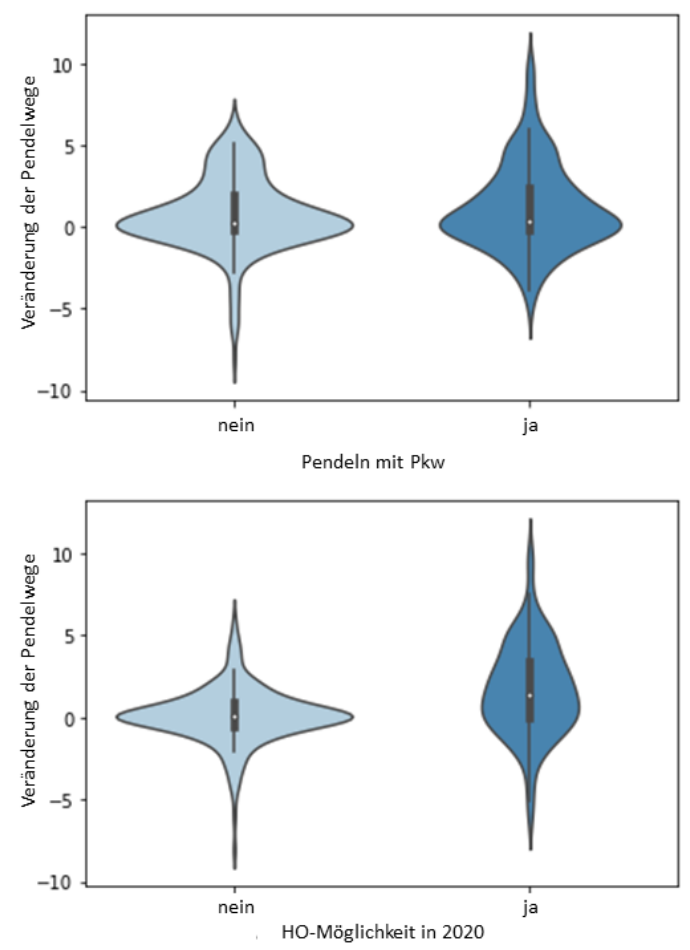
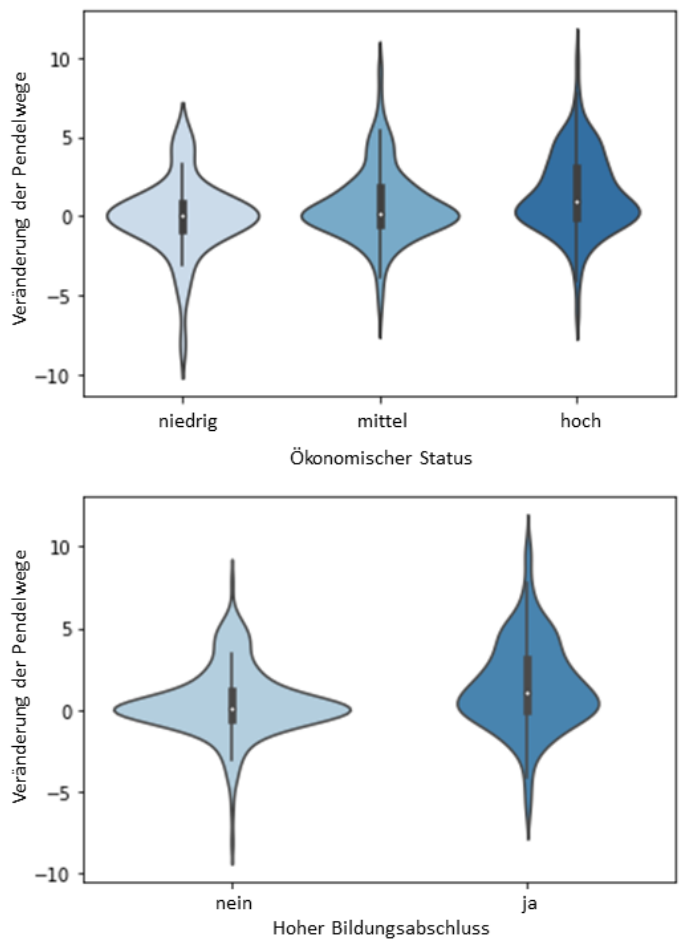
TABELLE 2 Ergebnisse der multiplen linearen Regression

\begin{tabular}{lrrrr}
\hline \hline Parameter & Schätzung & StandardFehler & t Wert & $\operatorname{Pr}>|\mathbf{t}|$ \\
\hline Intercept & 1.3634 & 0.2406 & 5.67 & $<.0001$ \\
\hline Gesamtpendelstrecke 2019 & 0.0045 & 0.0015 & 2.99 & 0.0030 \\
\hline Pendeln mit dem Auto - nein & 0.4450 & 0.2434 & 1.83 & 0.0682 \\
Pendeln mit dem Auto - ja & 0.0000 &. &. &. \\
\hline HO-Verfügbarkeit im Jahr 2020 - nein & -1.1437 & 0.2420 & -4.73 & $<.0001$ \\
HO-Verfügbarkeit im Jahr 2020 - ja & 0.0000 & &. & \\
\hline Hochgebildet - nein & -0.3971 & 0.2419 & -1.64 & 0.1014 \\
Hochgebildet - ja & 0.0000 & &. & \\
\hline Ökonomsicher Status - niedrig & -0.5966 & 0.4079 & -1.46 & 0.1443 \\
Ökonomsicher Status - mittel & -0.2059 & 0.2366 & -0.87 & 0.3846 \\
Ökonomsicher Status - hoch & 0.0000 & &. &. \\
\hline Beobachtungen & $\mathrm{N}=498$ & & & \\
R-Quadrat & 0.1418 & & & \\
F Wert & 11.84 & & & \\
Pr $>$ F & $<.0001$ & & & \\
\hline \hline
\end{tabular}

Eine positiver Parameterwert bedeutet, dass die Zahl der Wege zur Arbeit im Jahr 2020 im Vergleich zu 2019 verringert wurde. Die Gesamtpendeldistanz im Jahr 2019 beschreibt die Summe der Entfernungen, die in der gemeldeten Woche im Jahr 2019 zur Arbeit zurückgelegt wurden. Je höher die Summe der im Jahr 2019 zurückgelegten Entfernungen ist, desto wahrscheinlicher ist es, dass die Personen die Anzahl der Wege zur Arbeit im Jahr 2020 reduzierten. Darüber hinaus ist die Wahrscheinlichkeit, die Anzahl Wege zu reduzieren, höher für Personen, die nicht mit dem Auto zur Arbeit fahren.

Ein negativer Parameter deutet darauf hin, dass die Wege zur Arbeit im Jahr 2020 im Vergleich zu 2019 nicht reduziert werden. Die Ergebnisse zeigen, dass Personen, die keine Möglichkeit haben, im HO zu arbeiten, weniger wahrscheinlich ihre Wege zur Arbeit reduzieren. Dasselbe gilt für Personen mit einem niedrigeren ökonomischen Status im Vergleich zum höchsten ökonomischen Status sowie für Personen mit einem geringeren Bildungsgrad im Vergleich zu Personen mit einem Hochschulabschluss. Die letzten beiden Aspekte sind jedoch statistisch nicht signifikant.

Der R-Quadrat-Wert ist niedrig $(0,1418)$, was bedeutet, dass das Modell nur einen geringen Teil der Variabilität erklärt. Die Einbeziehung zusätzlicher Merkmale führte nicht zu einem höheren R-Quadrat. Andere soziodemografische Merkmale wie Geschlecht, Alter, Kinder im Haushalt und Haushaltsgröße verbesserten das Modell nicht und für sie wurden keine signifikanten Zusammenhänge mit der Veränderung der Zahl der Wege zur Arbeit festgestellt. Dies deutet darauf hin, dass die Verringerung der Wege zur Arbeit eher unabhängig von der Haushaltszusammensetzung und den soziodemografischen Merkmalen ist. Insgesamt besteht ein hochsignifikanter Zusammenhang zwischen der Möglichkeit, von zu Hause zu arbeiten, und der Veränderung der Anzahl der Wege sowie den Merkmalen des Pendelns (Gesamtpendelstrecke im Jahr 2019 und Pendeln mit dem Auto).

\section{Logit-Modell über die Möglichkeit, von Zuhause zu arbeiten}

Zur Analyse des HO-Verhaltens und des Einflusses der Pandemie haben wir mehrere Logit-Modelle geschätzt. Alle Modelle wurden mit dem R-Paket Apollo, Version 0.2.4 (Hess and Palma, 2021, 2019) geschätzt. Während der Schätzung wurde deutlich, dass die soziodemografischen Daten keinen Einfluss darauf haben, ob eine Person sich für $\mathrm{HO}$ entscheidet, sondern eher darauf, ob sie überhaupt von zu Hause arbeiten kann. Daher haben wir zunächst ein Logit-Modell geschätzt, um festzustellen, welche persönlichen und Haushaltsmerkmale die Möglichkeit beeinflusst, dass eine Person im $\mathrm{HO}$ arbeiten kann. Wie in der Literatur beschrieben, haben vor allem Berufstätige und Personen mit hohem Bildungsstand die Möglichkeit, von zu Hause aus zu arbeiten, daher haben wir Variablen einbezogen, die diese Umstände beschreiben. Der lineare Prädiktor $\mathrm{O}$, der zur Schätzung des Modells für die Möglichkeit, von zu Hause aus zu arbeiten, verwendet wurde, kann wie folgt ausgedrückt werden: 


$$
O_{n, t}=a s c+\beta_{\text {gender }} \text { Gender }_{n}+\beta_{\text {educ }} \text { Degree }_{n}+\beta_{E C S} E_{C S}, t+\beta_{\text {covid }} \text { Covid }
$$

wobei die Zeiträume $\mathrm{t}$ dem Erhebungszeitraum der Beobachtung entsprechen (d. h. 2018, 2019 oder 2020). Die Ausprägungen der einzelnen Variablen sind in Tabelle 3 aufgeführt. Der Parameter für COVID-19 berücksichtigt nur den Erhebungszeitraum und nicht verschiedene politische Maßnahmen, die während des Erhebungszeitraums durchgeführt wurden. Da wir für die Schätzung Längsschnittdaten mit wiederholten Wahlentscheidungen für dieselbe Person verwenden, multiplizieren wir die Wahrscheinlichkeiten über die Wahlbeobachtungen gleicher Befragten. Die Ergebnisse der Schätzung sind in Tabelle 3 dargestellt. Die für dieses Modell verwendeten Beobachtungen stammen von Befragten, die im Jahr 2020 und mindestens im Jahr 2018 oder 2019 Angaben gemacht haben, was zu einem Datensatz mit 2.117 Beobachtungen führt.

Die Schätzungen zeigen, dass Frauen und Personen mit geringerem Bildungsniveau seltener in der Lage sind, von zu Hause zu arbeiten. Personen ohne Abschluss (oder mit niedrigem Schulabschluss) sind weniger wahrscheinlich in der Lage, von zu Hause zu arbeiten, als Personen mit Abitur oder mit einem Hochschulabschluss. Die Schätzungen zeigen auch, dass je höher der ökonomische Status ist, desto wahrscheinlicher ist es, dass die Personen im $\mathrm{HO}$ arbeiten können. Dies ist nicht überraschend, da diese beruflichen Tätigkeiten auch mit einem höheren Einkommen verbunden sind. Die COVID-19-Pandemie wirkt sich positiv auf den linearen Prädiktor aus, was bedeutet, dass die Pandemie einen positiven Einfluss auf die Möglichkeit zur HO-Nutzung hatte.

\section{Mixed-Logit-Modell zur Entscheidung für HO-Nutzung}

Zur Analyse der Auswirkungen der Pandemie auf die Entscheidung der Befragten, $\mathrm{HO}$ zu nutzen, wenn sie die Möglichkeit dazu haben, haben wir auch ein Logit-Modell ge-

TABELLE 3 Variablen und Ergebnisse des Logit-Modells für die Möglichkeit, von Zuhause aus zu arbeiten

\begin{tabular}{|c|c|c|c|c|}
\hline variabel & $\begin{array}{l}\text { Name des Parameters in der } \\
\text { Funktion (1) }\end{array}$ & Ausprägung & Parameterwert & $\begin{array}{c}\text { robuste t- } \\
\text { Quote }\end{array}$ \\
\hline \multirow[b]{2}{*}{$\begin{array}{l}\text { Alternative spezifische } \\
\text { Konstante }\end{array}$} & \multirow[b]{2}{*}{ asc } & Arbeit von Zuhause möglich & -2.4259 & -8.849 \\
\hline & & $\begin{array}{l}\text { Arbeit von Zuhause nicht möglich } \\
\text { (Referenz) }\end{array}$ & - & - \\
\hline \multirow{2}{*}{ Geschlecht } & \multirow{2}{*}{$\beta_{\text {Geschlecht }}$} & männlich (Referenz) & - & - \\
\hline & & weiblich & -0.5099 & -3.821 \\
\hline \multirow{3}{*}{$\begin{array}{l}\text { höchster } \\
\text { Bildungsabschluss }\end{array}$} & \multirow{3}{*}{$\beta_{e d u c}$} & kein Abschluss (Referenz) & - & - \\
\hline & & Abitur & 0.9356 & 4.689 \\
\hline & & College- oder Universitätsabschluss & 1.7749 & 11.119 \\
\hline \multirow{3}{*}{$\begin{array}{l}\text { ökonomsicher Status } \\
(\mathrm{HH})\end{array}$} & \multirow{3}{*}{$\beta_{e c s}$} & niedrig (Referenz.) & - & - \\
\hline & & mittel & 0.5633 & 2.139 \\
\hline & & hoch & 1.0933 & 4.032 \\
\hline \multirow{2}{*}{ COVID-19 } & \multirow{2}{*}{$\beta_{\text {Covid }}$} & nein (2018 und 2019) (Referenz) & - & - \\
\hline & & ja $(2020)$ & 0.6998 & 7.767 \\
\hline Beobachtungen & $N=2,117$ & & & \\
\hline LL(Start) / LL(0) & -519.1672 & & & \\
\hline LL (endgültig) & -322.3423 & & & \\
\hline Rho-Quadrat & 0.3791 & & & \\
\hline
\end{tabular}

schätzt.

Für das Wahlmodell haben wir die Antworten bezüglich des HO-Verhaltens von vier Kategorien auf zwei umcodiert: In die Wahl für HO und die Wahl gegen $\mathrm{HO}$, während die Antwort "Diese Möglichkeit besteht nicht." in dem zuvor beschriebenen Modell zur Analyse der HO-Verfügbarkeit berücksichtigt wurde. Um herauszufinden, welche Variablen diese Wahl während der COVID-19-Pandemie anders beeinflussen als im Zustand davor, haben wir die Heterogenität zwischen den einzelnen Entscheidungen berücksichtigt, indem wir ein Mixed-Logit-Modell geschätzt haben (Train, 2009). Wir haben mehrere Modelle geschätzt, um herauszufinden, welche Attribute die Wahl signifikant beeinflussen und wo Heterogenität zwischen den einzelnen Möglichkeiten vorhanden ist. Das Modell berücksichtigt nur die intrapersonelle Heterogenität zwischen den einzelnen Entscheidungssituationen, nicht aber zwischen den einzelnen Personen, da wir die Auswirkungen der Pandemie auf die Wahlmöglichkeiten der 
einzelnen Personen analysieren wollen. Für die simulierte Log-Likelihood-Berechnung haben wir 750 Halton-Draws verwendet. Die Nutzenfunktion für Heimarbeit, die im endgültigen Modell für die Person n zum Zeitpunkt t verwendet

$$
V_{n, t}=\operatorname{asc}+\beta_{\text {commute }} C D_{n, t}+\beta_{\text {workloc }} W L_{n, t}+\beta_{k i d s} K I H_{n, t}+\beta_{\text {single }} S H H_{n, t}+\beta_{\text {Covid }} \text { Covid }_{n, t}
$$

wird, ist gegeben durch:

wobei asc die alternativspezifische Konstante ist, CD die Pendeldistanz in Kilometern, WL ist ein Attribut zur Beschreibung der Lage des Arbeitsplatzes, wobei WL 1 ist, wenn der Arbeitsplatz am Stadtrand liegt und 0 in den sonstigen Fällen. Die Originaldaten enthalten Informationen über die Lage des Arbeitsplatzes auf fünf verschiedenen Ebenen, die Variable war jedoch nur für eine Attributsebene signifikant. $\mathrm{KIH}$ ist binär und ist 1, wenn es im Haushalt Kinder unter 10 Jahren gibt. COVID ist ebenfalls eine binäre Variable, die 1 ist, wenn der Erhebungszeitraum 2020 war, um die direkten
Auswirkungen der Pandemie auf die Entscheidung zur HONutzung zu berücksichtigen. SHH berücksichtigt, dass $\mathrm{HO}$ für alleinlebende Befragte anders bewerten als Personen, die mit anderen zusammenleben. Dies war auch die einzige Variable, die signifikante Heterogenität aufwies. Das MNLModell ohne Mixing wurde durch das Modell, das Heterogenität zulässt, verworfen. Die Heterogenität wurde mit Hilfe der von Fosgerau und Mabit vorgestellten Potenzreihenerweiterung berücksichtigt (Fosgerau and Mabit, 2013). Das endgültige Modell enthielt einen Mixing-Koeffizienten für Einzelhaushalte, gegeben durch:

$$
\beta_{\text {single }}=\beta_{\text {single }}^{0}+\sigma_{\text {single }, 1} u_{n}+\sigma_{\text {single }, 2}\left(u_{n}\right)^{2}
$$

\begin{tabular}{|c|c|c|c|c|}
\hline Variable & $\begin{array}{l}\text { Parametername in den } \\
\text { Funktionen (2) \& (3) }\end{array}$ & Attributsausprägungen & Parameterwert & $\begin{array}{l}\text { robuste } \\
\text { t-Wert }\end{array}$ \\
\hline \multirow{2}{*}{$\begin{array}{l}\text { Alternative spezifische } \\
\text { Konstante }\end{array}$} & \multirow[b]{2}{*}{ asc } & Arbeiten von Zuhause & $-24,259$ & $-8,849$ \\
\hline & & $\begin{array}{l}\text { Kein Arbeiten von Zuhause } \\
\text { (konst.) }\end{array}$ & - & - \\
\hline $\begin{array}{l}\text { Entfernung zum } \\
\text { Arbeitsplatz }\end{array}$ & B_commute & Kilometer & 0.02012 & 3,162 \\
\hline \multirow{2}{*}{ Ort des Arbeitsplatzes } & \multirow{2}{*}{ B_workloc } & Sonstiges (Konst.) & - & - \\
\hline & & Außenbezirke einer Stadt & -0.89224 & $-2,852$ \\
\hline \multirow{2}{*}{$\begin{array}{l}\text { Kinder unter } 10 \text { Jahren in } \\
\mathrm{HH}\end{array}$} & \multirow{2}{*}{$\beta_{-}$kids } & nein (Konst.) & - & - \\
\hline & & ja & 0.5633 & 2,139 \\
\hline \multirow{3}{*}{ Single-Haushalt } & B_kids & & 0.02994 & 1,139 \\
\hline & $\sigma_{\text {single, } 1}$ & $\mathrm{HH}-\mathrm{Größe}=1$ & -0.02215 & $-1,465$ \\
\hline & $\sigma_{\text {single }, 2}$ & & -0.59413 & $-2,124$ \\
\hline \multirow{2}{*}{ COVID-19 } & \multirow{2}{*}{$\beta_{-}$COVID } & nein (2018 und 2019) (konst.) & - & - \\
\hline & & ja (2020) & 107,853 & 4,788 \\
\hline Beobachtungen & 2.117 & & & \\
\hline $\mathrm{LL}($ Start $) / \mathrm{LL}(0)$ & $-1,453.53$ & & & \\
\hline LL (endgültig) & $-1,149.469$ & & & \\
\hline Rho-Quadrat & 0.3791 & & & \\
\hline
\end{tabular}

Tabelle 4 Variablen und Ergebnisse des gemischten Logit-Modells zur Entscheidung für das Arbeiten von Zuhause

Die Ergebnisse des geschätzten gemischten Logit-Modells sind in Tabelle 4 dargestellt. Für die Schätzung des gemischten Logit-Modells wurde derselbe Datensatz wie für das zuvor beschriebene Modell verwendet.

Die Parameterschätzungen zeigen, dass die Pendeldistanz die Entscheidung für Heimarbeit positiv beeinflusst, d. h. Befragte mit längeren Pendelwegen arbeiten eher von zu Hause aus. Der Parameter selbst ist im Vergleich zu den anderen Parametern relativ klein, was auf das Ausmaß der Pendelent- fernung im Vergleich zu den anderen Variablen zurückzuführen ist, die alle mit dummy-codiert versehen sind. Was den Arbeitsplatz der Befragten betrifft, so hatten nur diejenigen, die am Rande einer Stadt wohnen, einen signifikanten Einfluss auf die Wahl. Das lässt vermuten, dass die relativ gute Erreichbarkeit solcher Arbeitsplätze dazu führt, dass die Befragten nicht aufgrund der Erreichbarkeit des Arbeitsplatzes in $\mathrm{HO}$ gehen müssen, da das Pendeln als akzeptabel angesehen wird. Der Parameter für im Haushalt lebende Kinder unter zehn Jahren ist erwartungsgemäß positiv. Befragte, die 
Kinder zu betreuuen haben, profitieren von der Arbeit von zu Hause aus, insbesondere wenn z.B. das Kind krank ist und keine andere regelmäßige Betreuung zur Verfügung steht. Der Parameter bezüglich des Einflusses der Pandemie ist erwartungsgemäß positiv. Unter sonst gleichen Bedingungen hat allein die Pandemie die Wahrscheinlichkeit erhöht, dass die Befragten von zu Hause arbeiten, was bedeutet, dass die Befragten aufgrund der Pandemie eher die Chance ergriffen haben, von zu Hause aus zu arbeiten. Die Parameter für Einpersonenhaushalte, d. h. alleinlebende Personen, zeigen, dass sich diese Befragten eher nicht für die Heimarbeit entscheiden, aber die Heterogenität deutet darauf hin, dass das Alleinleben während der Pandemie nicht so stark ins Gewicht fiel wie zuvor.

\section{Diskussion}

Unsere Ergebnisse stimmen mit denen in der Literatur überein. Die Arbeit von zu Hause aus reduziert eindeutig die Pendeldistanzen und die Anzahl der Pendelwege. Die Ergebnisse des linearen Regressionsmodells zeigen, dass Personen, die die Möglichkeit haben, von zu Hause aus zu arbeiten, ihre Pendelwege deutlich reduzieren, was darauf hindeutet, dass die Befragten die Möglichkeit nutzen, regelmäßig von zu Hause aus zu arbeiten. Während die Zahl der Personen, die von zu Hause arbeiten, zunahm, blieben die Faktoren, die dafür ausschlaggebend sind, gleich. Heimarbeit ist nach wie vor ein Privileg hoch gebildeter Männer mit hohem ökonomischem Status, auch wenn aufgrund der COVID-19-Pandemie mehr Menschen die Möglichkeit erhielten, von zu Hause aus zu arbeiten. Unsere Ergebnisse zeigen, dass die Koeffizienten, die die Entscheidung für Heimarbeit charakterisieren, gleichgeblieben sind, so dass wir davon ausgehen können, dass Personen, die aufgrund der Pandemie die Möglichkeit hatten ins $\mathrm{HO}$ zu gehen, auch weiterhin von zu Hause arbeiten wollen, wenn sie die Gelegenheit dazu haben. Eine Ausnahme bilden die Alleinlebenden: Während der Pandemie wurde das Risiko einer Ansteckung teilweise höher bewertet als das Bedürfnis nach sozialen Kontakten. Diese Personen werden nach der Pandemie am ehesten wieder vor Ort arbeiten und nicht so häufig das $\mathrm{HO}$ nutzen. Bisherige Studien wurden kurzfristig während der Pandemie entwickelt, wobei die Situation vor COVID-19 oft nur retrospektiv erfasst wurde und daher ungenau oder verzerrt sein kann. Das MOP ermöglicht es uns dagegen, die bereits 2018 und 2019 gemeldeten unverzerrten Daten zu verwenden und sie mit den Daten der gleichen Personen aus dem Jahr 2020 in Beziehung zu setzen.

\section{FAZIT}

In diesem Paper wird eine Analyse der Auswirkungen von $\mathrm{HO}$ und der Einflüsse auf die Entscheidung, von zu Hause aus zu arbeiten, vorgestellt. Dank des Paneldesigns liefert das Deutsche Mobilitätspanel einzigartige Daten von Personen, die vor und während der COVID-19-Pandemie teilgenommen haben. Die Daten ermöglichen es somit, die durch die Pandemie ausgelösten Verhaltensänderungen zu identifizieren. Die Ergebnisse zeigen, dass während der Pandemie der Anteil der Personen, die von zu Hause arbeiteten, stark zunahm und somit das Pendeln zur Arbeit folglich abnahm. Personen mit hohem Bildungsstatus, die auch einen hohen ökonomischen Status haben, arbeiten eher von zu Hause aus. Dies führt zu einer Diskussion über die soziale Gerechtigkeit: Personen mit einem hohen ökonomischen Status sind in der Regel in einer Position mit großer Autonomie und haben ein hohes Einkommen, was ihnen eher die Möglichkeit gibt, zu Hause zu arbeiten. Im Gegensatz dazu haben Menschen, die z. B. im verarbeitenden Gewerbe arbeiten und in der Regel ein geringeres Gehalt beziehen, keine Möglichkeit zur Heimarbeit und damit ein höheres Infektionsrisiko bzw. müssen sich beurlauben lassen oder sogar ihren Arbeitsplatz aufgeben, wenn sie das Infektionsrisiko verringern wollen. Das gilt nicht nur für das Infektionsrisiko am Arbeitsplatz, sondern auch beim Pendeln mit öffentlichen Verkehrsmitteln. Dieser Effekt wurde von Shakibaei et al. beschrieben. Die Ergebnisse dieser Studie zeigen, dass die hohen Treibstoffkosten in der Türkei Personen dazu zwangen, während der Pandemie mit öffentlichen Verkehrsmitteln zu pendeln, selbst wenn sie ein Auto besaßen (Shakibaei et al., 2021). Daraus sollten die politischen EntscheidungsträgerInnen zwei Schlussfolgerungen ziehen: Erstens sollte die Möglichkeit, von zu Hause zu arbeiten, wo immer möglich, eingeführt werden. Zweitens sollte Personen, deren Tätigkeit HO-Nutzung nicht ermöglicht, der bestmögliche Schutz vor Infektionen geboten werden. So kann beispielsweise ein großes Angebot an öffentlichen Verkehrsmitteln dazu beitragen, die Exposition während des Pendelns zu minimieren.

Für die Zeit nach der Pandemie wird die verstärkte Nutzung von $\mathrm{HO}$ als Maßnahme zur Verringerung der Verkehrsüberlastung im Verkehrssystem und zur Verringerung des gesamten Verkehrsaufkommens und damit der verkehrsbedingten Emissionen angesehen. In Anbetracht der bestehenden Ungleichheit in Bezug darauf, wer in der Lage ist, von zu Hause zu arbeiten, sollten politische Maßnahmen, die auf eine Ausweitung der Telearbeit abzielen, jedoch sorgfältig ausgewählt werden. Eine Anhebung der Pendelkosten als Maßnahme zur Förderung der Telearbeit (Handy and Mokhtarian, 1995) wird wahrscheinlich denjenigen schaden, die bereits einen Arbeitsplatz mit geringerem Einkommen haben und nicht in der Lage sind, HO-Regelungen zu nutzen (Felstead et al., 2002).

Neben dem Vorteil, dass Heimarbeit den Verkehr reduzieren kann, sollten auch die Nachteile von Telearbeit berücksichtigt werden. Unsere Ergebnisse zeigen, dass Menschen, die allein leben, sich eher gegen Arbeit im $\mathrm{HO}$ entscheiden. In 
diesem Zusammenhang sollte die Arbeit am Arbeitsplatz im Unternehmen auch als ein Ort für soziale Kontakte gesehen werden. Ein längerer Aufenthalt zu Hause kann für alleinlebende Menschen zu einer Belastung werden. Auch aus Sicht der ArbeitgeberInnen hat HO Nachteile: MitarbeiterInnen identifizieren sich evtl. weniger mit dem Unternehmen, der Teamgeist und die Solidarität können schwächer werden, wenn sich $\mathrm{HO}$ stärker durchsetzt. Telearbeit ist also nicht nur eine Entscheidung der Arbeitnehmerlnnen, sondern auch eine der ArbeitgeberInnen. Es ist absehbar, dass es in Zukunft auch hybride Fernarbeitslösungen geben wird, bei denen sowohl die ArbeitgeberInnen als auch die Arbeitnehmerlnnen von der Möglichkeit zur HO-Nutzung profitieren, aber dennoch die Vorteile der Arbeit vor Ort von Zeit zu Zeit beibehalten.

Insgesamt kann die COVID-19-Pandemie als ein bedeutendes Ereignis angesehen werden, das die Menschen dazu veranlasst hat, mehr $\mathrm{HO}$ zu nutzen, auch wenn sie dies vorher nicht getan haben. Normalerweise ist das Verhalten stabil und lässt sich nicht leicht anpassen (Gärling and Axhausen, 2003). Durch die abrupte Verhaltensänderung, die durch die Pandemie verursacht wurde, waren die Menschen jedoch gezwungen, zu lernen, ihre Gewohnheiten aufzugeben. Dies eröffnet den Menschen eine Chance, ihr Verhalten zu überdenken. Unsere Ergebnisse unterstreichen dies, da die Mehrheit unserer Stichprobe $\mathrm{HO}$ generell positiv bewertet. Wir gehen jedoch auch davon aus, dass Personen, die Telearbeit und HO-Nutzung negativ bewerten, bald nach der Pandemie zu ihrem normalen Pendelverhalten zurückkehren werden.

\section{LITERATURVERZEICHNIS}

Beck, M.J., Hensher, D.A., Wei, E., 2020. Slowly coming out of COVID-19 restrictions in Australia: Implications for working from home and commuting trips by car and public transport. Journal of Transport Geography 88, 102846. https://doi.org/10.1016/j.jtrangeo.2020.102846.

Clear, F., Dickson, K., 2005. Teleworking practice in small and medium-sized firms: management style and worker autonomy. New Technology, Work and Employment 20, 218233. https://doi.org/10.1111/j.1468-005X.2005.00155.x.

de Haas, M., Faber, R., Hamersma, M., 2020. How COVID-19 and the Dutch 'intelligent lockdown' change activities, work and travel behaviour: Evidence from longitudinal data in the Netherlands. Transportation Research Interdisciplinary Perspectives 6, 100150. https://doi.org/10.1016/j. trip.2020.100150.

Destatis, 2019. Bevölkerung, Erwerbstätige, Erwerbslose: Deutschland, Jahre, Geschlecht, Beschäftigungsumfang, Altersgruppen.

Ecke, L., Chlond, B., Magdolen, M., Vortisch, P., 2020. Deutsches Mobilitätspanel (MOP) - Wissenschaftliche Begleitung und Auswertungen Bericht 2019/2020: Alltagsmobilität und Fahrleistung. https://doi.org/10.5445/IR/1000126557.

European Foundation for the Improvement of Living and Working Conditions (Ed.), 2018. Eurofound yearbook 2017 Living and working in Europe. Eurofound yearbook.

European Foundation for the Improvement of Living and Working Conditions (Ed.), 2010. Telework in the European Union.

Felstead, A., Jewson, N., Phizacklea, A., Walters, S., 2002. The option to work at home: another privilege for the favoured few? New Technology, Work and Employment 17, 204-223. https://doi.org/10.1111/1468-005X.00105.

Fosgerau, M., Mabit, S.L., 2013. Easy and flexible mixture distributions. Economics Letters 120, 206-210. https://doi. org/10.1016/j.econlet.2013.03.050.

Gärling, T., Axhausen, K.W., 2003. Introduction: Habitual travel choice. Transportation 30, 1-11. https://doi. org/10.1023/A:1021230223001.

Handy, S.L., Mokhtarian, P.L., 1995. Planning for Telecommuting Measurement and Policy Issues. Journal of the American Planning Association 61, 99-111. https://doi. org/10.1080/01944369508975623. 
Hess, S., Palma, D., 2021. Apollo version 0.2.4, user manual.

Hess, S., Palma, D., 2019. Apollo: A flexible, powerful and customisable freeware package for choice model estimation and application. Journal of Choice Modelling 32, 100170. https://doi.org/10.1016/j.jocm.2019.100170.

Hiselius, L.W., Arnfalk, P., 2021. When the impossible becomes possible: COVID-19's impact on work and travel patterns in Swedish public agencies. European Transport Research Review 13. https://doi.org/10.1186/s12544-02100471-9.

Kolarova, V., Eisenmann, C., Nobis, C., Winkler, C., Lenz, B., 2021. Analysing the impact of the COVID-19 outbreak on everyday travel behaviour in Germany and potential implications for future travel patterns. European Transport Research Review 13. https://doi.org/10.1186/s12544-021-00486-2.

Mokhtarian, P.L., Salomon, I., 1996a. Modeling the choice of telecommuting: 2 . A case of the preferred impossible alternative. Environment and Planning 28, 1859-1876.

Mokhtarian, P.L., Salomon, I., 1996b. Modeling the Choice of Telecommuting 3: Modelling the Choice of Telecommuting 3: Identifying the Choice Set and Estimating Binary Models for Technology-Based Alternatives. Environment and Planning 28, 1877-1894.

Molloy, J., Schatzmann, T., Schoeman, B., Tchervenkov, C., Hintermann, B., Axhausen, K.W., 2021. Observed impacts of the Covid-19 first wave on travel behaviour in Switzerland based on a large GPS panel. Transport Policy 104, 43-51. https://doi.org/10.1016/j.tranpol.2021.01.009.

Shakibaei, S., de Jong, G.C., Alpkökin, P., Rashidi, T.H., 2021. Impact of the COVID-19 pandemic on travel behavior in Istanbul: A panel data analysis. Sustainable cities and society 65. https://doi.org/10.1016/j.scs.2020.102619.

Shamshiripour, A., Rahimi, E., Shabanpour, R., Mohammadian, A., 2020. How is COVID-19 reshaping activity-travel behavior? Evidence from a comprehensive survey in Chicago. Transportation Research Interdisciplinary Perspectives 7, 100216. https://doi.org/10.1016/j.trip.2020.100216.

Train, K.E., 2009. Discrete Choice Methods with Simulation. Cambridge University Press, Cambridge. https://doi. org/10.1017/CBO9780511805271.

WHO, 2020. Listings of WHO's response to COVID-19.

Zumkeller, D., Chlond, B., 2009. Dynamics of Change: Fifteen-Year German Mobility Panel, in: Transportation Re- search Board (Ed.), TRB 88th Annual Meeting Compendium of Papers.

\section{AutorInnenangaben}

\section{Anna Reiffer}

Institut für Verkehrswesen, Karlsruher Institut für Technologie (KIT), Kaiserstr. 12, 76131 Karlsruhe, Deutschland

E-Mail: anna.reiffer@kit.edu

\section{Miriam Magdolen}

Institut für Verkehrswesen, Karlsruher Institut für Technologie (KIT), Kaiserstr. 12, 76131 Karlsruhe, Deutschland

E-Mail: miriam.magdolen@kit.edu

\section{Lisa Ecke}

Institut für Verkehrswesen, Karlsruher Institut für Technologie (KIT), Kaiserstr. 12, 76131 Karlsruhe, Deutschland

E-Mail: lisa.ecke@kit.edu

\section{Peter Vortisch}

Institut für Verkehrswesen, Karlsruher Institut für Technologie (KIT), Kaiserstr. 12, 76131 Karlsruhe, Deutschland

E-Mail: peter.vortisch@kit.edu 